\title{
Posicionamiento de la familia ante el uso de la tableta en el aprendizaje del alumnado de Educación Primaria
}

\author{
Cristina Sánchez-Martínez \\ c.sanchez@uvigo.es \\ María-Carmen Ricoy \\ cricoy@uvigo.es \\ Universidad de Vigo, España
}

\begin{abstract}
Resumen
La influenciada de las TIC es notoria en todos los ámbitos y su utilización también se lleva incrementado en el contexto familiar y escolar. Por ello, el objetivo central de este trabajo es analizar y conocer el posicionamiento de la familia, concretamente de los padresmadres, en la integración de la tableta digital en el alumnado de Educación Primaria. La presente investigación se encuadra en la metodología cualitativa, para ello se ha abordado un estudio de casos múltiples, a partir de la recogida de narrativas a los progenitores del alumnado de cuatro centros educativos situados en el noroeste de España. A los datos obtenidos se le aplicó un análisis de contenido. Como principales resultados y conclusiones cabe señalar que los padres-madres ponen de manifiesto la inexistencia de dificultades para trabajar con la tableta con sus descendientes, aunque no recibieron formación específica ni la consideran necesaria. Su valoración en general es positiva y sostienen que la utilización de la tableta aumenta la motivación de sus hijos-as, sin embargo no encuentran mejora en su rendimiento escolar. Es de destacar que acompañan a sus hijosas, a petición de los mismos, con el uso de la tableta en el hogar sin estimularles directamente.
\end{abstract}

\section{Keywords}

Familia; contexto escolar; contexto familiar; educación primaria; TIC; tableta digital. 


\title{
Position of the family towards tablet use on learning of Primary Education students
}

\section{Cristina Sánchez-Martínez}

c.sanchez@uvigo.es

\section{María-Carmen Ricoy}

cricoy@uvigo.es

University of Vigo, Spain

\begin{abstract}
Information and Communication Technologies influence is becoming more and more evident in all areas and its use has also increased in family and school context. Therefore, the main objective of this work is to analyze and to know family positioning and involvement, specifically of the parents, on the integration of the digital tablet in Primary Education students. The present investigation is part of the qualitative methodology, for it has approached a study of multiple cases, from the collection of parents narratives of students from four educational centers located at northwest of Spain. A content analysis was applied to obtained data. As main results and conclusions, it should be pointed out that fathers and mothers show that there are no difficulties in working with the tablet with their descendants, although they did not receive any specific training or consider it necessary. Their overall assessment is positive and they argue that tablet use increases their children motivation, but they do not find improvement in their school performance. It is noteworthy that they keep track of children, upon children request, on tablet use at home without stimulating them directly.
\end{abstract}

\section{Keywords}

Family; school context; family context; primary education; ICT; digital tablet. 


\section{Introducción}

El contexto mundial actual se caracteriza, entre otros, por las posibilidades que tiene de producir y difundir con gran rapidez la información, así como por compartirla de modo instantáneo. Fue a partir de la proliferación de Internet, en los años 90, cuando se plantean nuevos espacios digitales en los que el usuario consume información, produce contenidos y se convierte en beneficiario (Campos-Domínguez y Redondo-García, 2015). Ante este panorama también cabe señalar que las nuevas generaciones forman parte de los llamados nativos digitales. Estos cuentan con una serie de herramientas tecnológicas a su alcance que les han permitido empoderarse de una forma genuina para recibir, crear y difundir contenidos (Espiritusanto, 2017). En este sentido, este colectivo puede ejecutar múltiples tareas de forma momentánea y cuenta con habilidades naturales para el uso de las Tecnologías de la Información y Comunicación (TIC), aspectos que les diferenciaran de sus progenitores. Utilizan las TIC de forma cotidiana para jugar, escuchar música, obtener información y también como apoyo para realizar sus tareas escolares (López-González y Anaya, 2016; Hernández, 2017). En este contexto, la escuela y la familia tienen un papel fundamental y han de coordinar/supervisar e incentivar oportunamente el proceso de empoderamiento a partir del uso de la tecnología en los más jóvenes. Para Hargreaves (2012), los ajustes y la consecución de los cambios derivados de los medios digitales son imprescindibles para situar al alumnado en la sociedad actual.

La familia y la escuela, como instituciones milenarias de peso, continúan siendo ámbitos esenciales de influencia para el desarrollo y la educación de los más pequeños. La familia es, por excelencia, el primer agente de socialización, por ello juega un papel primordial contribuyendo al desarrollo físico, emocional, intelectual y social de los más pequeños. Asimismo los padres y madres, cada vez, en mayor medida, tienen una función relevante en la educación mediática de sus hijos-as. De modo que, es esencial que conozcan y participen en la formación digital de sus hijos-as para poder prestarles apoyo, orientación y asesoramiento en los trabajos que realicen en casa (Martín et al., 2009; Ferrer, 2017). En este sentido, siguiendo a Fernández-Rodrigo y Carrera (2016), las propias familias consideran que es importante que estén asesoradas sobre la implementación de la tableta digital en el aula de sus hijos-as. Cabe resaltar que a los padres y madres del siglo XXI se le plantea un reto sin precedentes para participar en la educación digital de sus descendientes (Livingstone, Haddon, Görzig y Ólafsson, 2011; Urías-Martínez, Urías-Murrieta y Valdés-Cuervo, 2017). Además, es usual que las habilidades técnicas, para el manejo de los aparatos digitales, de los más pequeños superen a las de muchas personas adultas, entre las que se incluyen los progenitores y el profesorado. De este modo pueden encontrarse en situaciones difíciles o sentir dificultada su labor. En este sentido, tanto el papel de los padres-madres, como el del profesorado es más complejo que nunca, ya que les exige una gran implicación (Aguilar y Leiva, 2012).

Un aspecto a tener en cuenta, en la Sociedad de la Información, es la existencia de brechas digitales que podrían derivar, tanto las dificultades desencadenadas por el nivel adquisitivo de las familias, como por los obstáculos encontrados digitalmente. De este modo, se manifiestan diferentes desigualdades que emanan de la brecha digital. Es obvio que no todas las familias se pueden permitir el acceso a Internet en el hogar, contar con dispositivos (como ordenadores, tabletas, etc.). García, Ferrás, Rocha y Aguilera (2016) y Esteban-Moreno (2017) afirman que existe una brecha digital que descarta inevitablemente a aquellas personas que están desconectadas o que no cuentan con dispositivos tecnológicos; asimismo alude a una brecha del conocimiento que arrincona a las que no están conectadas o no utilización las herramientas digitales. Sin lugar a duda, la brecha generada entre distintos grupos de edad, colectivos o áreas geográficas que repercute directamente en la cantidad y calidad del elenco de actividades desarrolladas.

En la línea de lo adelantado, cabe incidir en la importancia que tiene la relación del binomio familia- 
escuela, especialmente en una etapa, de especial dependencia y que exige gran protección, como es la de la infancia. En este periodo vital el papel de la familia, en particular de las figuras materna y paterna, presenta una gran importancia. Con todo, siguiendo a Cabrera (2009), no podemos olvidar que la educación es una tarea compartida entre padres/madres y educadores/as. Por este motivo es necesario que la familia y la escuela estén en contacto y se coordinen (Morgado y Román, 2011).

Las propuestas holísticas e integradoras de intervención socio-educativa, que tengan en cuenta el entorno actual, se han de caracterizar por el uso de estrategias comunicativas, de apertura, flexibilidad, comprensión y continuidad para establecer lazos sólidos entre los progenitores y el contexto escolar. No obstante, para conocer las necesidades y expectativas de la familia pueden resultar de gran utilidad las escuelas de padres y madres, o como foco de iniciativas, centrándose en estrategias preventivas la institución escolar ha de ejercer un papel activo y enriquecedor que repercuta positivamente en la comunidad o en el contexto social (Ricoy y Feliz, 2002). En diferentes estudios (Lozano, Alaraz y Colás, 2013; Collet y Tort, 2013; García-Rodríguez y Paíno, 2017) se ha puesto de manifiesto la importancia del acercamiento de la cultura escolar y la familiar. Además, cabe destacar que la introducción de las TIC en los centros escolares supone una vía para mejorar las relaciones y la comunicación entre la familia y la escuela, aproximando ambas culturas (Sánchez y Cortada, 2015). Como no podría ser de otro modo, también los padres y las madres están inmersos en la cultura digital que rodea a sus pequeños-as y esto le permite compartir con ellos diversas experiencias.

Las TIC cuentan con un protagonismo fundamental en la sociedad actual, su expansión al ámbito educativo es una realidad y posibilita múltiples alternativas didácticas (Arias-Ortiz y Cristia, 2014; Cortés, Méndez y Lacasa, 2016; Tuzel y Hobbs, 2017). Cada vez en mayor medida, los centros educativos integran la tecnología implantando distintas experiencias curriculares con diversos dispositivos. En este sentido, en particular en el contexto español, cabe referir las iniciativas que se están implementando con el uso de la tableta digital. De hecho, siguiendo a Feliz-Murias, Ricoy y Feliz (2014) la tableta es uno de los dispositivos en boga en las escuelas y universidades. Asimismo, su potencial se acrecienta cuando se cuenta con experiencias diversificadas en el contexto del hogar (Mascheroni y Kjartan, 2014). Es por ello, de gran interés abordar estudios sobre la inclusión curricular de la tableta y el papel de la familia ya que, por el momento, todavía existen pocos hallazgos que hayan profundizado en sus posibilidades educativas (Sánchez-Martínez y Ricoy, 2015).

Las tecnologías han penetrado en los hogares españoles, como así se ha venido reflejando en recientes estudios e informes. Por ejemplo, el Instituto Nacional de Estadística (INE, 2017) en una encuesta sobre equipamiento y uso de TIC en los hogares españoles, ofrece datos sorprendentes sobre la presencia de la conexión a Internet en las viviendas españolas. En este sentido el 83,4\% de los hogares cuentan con conexión a internet y el $84,6 \%$ de las personas han usado internet en los últimos 3 meses. En cuanto al equipamiento de las viviendas en algunos productos de TIC correspondiente a los años 2016 y 2017, cabe destacar que se indaga por primera vez la disposición de la tableta digital. En la actualidad a tenor de este estudio del INE la tableta se encuentra implantada en más de la mitad $(52,4 \%)$ de los hogares españoles. Llama poderosamente la atención el posicionamiento conseguido por este dispositivo, al tratarse de un recurso que se utiliza fundamentalmente para el ocio. Por supuesto el teléfono móvil ya está muy próximo al $100 \%$, a la altura del ordenador.

Ante los cambios producidos en la Sociedad de la Información y de la Comunicación el proceso formativo exige transformaciones acuciantes para mejorarlo. Por ello, en la etapa de la Educación Primaria es fundamental que, tanto el profesorado, como los padres y madres tomen un papel activo y dialogante para compartir y apoyar a las nuevas generaciones de nativos digitales a usar 
con propiedad y prudencia los recursos digitales, entre ellos la tableta digital, también como medios para el aprendizaje. En ese sentido, la finalidad principal de este estudio es delimitar y analizar la posición de la familia ante la inclusión de la tableta digital en el aprendizaje de los hijos e/o hijas que cursan la Educación Primaria. Como objetivos específicos del estudio se han planteado los siguientes:

- Descubrir las inquietudes producidas por los primeros contactos de las familias con la tableta.

- Retratar el sentir de la familia sobre la inclusión de la tableta en el aprendizaje.

- Determinar la implicación de la familia ante el uso que realizan de la tableta sus hijosas.

\section{Metodología}

Este trabajo se enmarca en la investigación cualitativa, desde el enfoque narrativo, que es de utilidad para realizar estudios de forma contextualizada, atendiendo primordialmente al entorno en el que se desarrollan. Siguiendo a Balcázar, González, Gurrola y Moysén (2015) este tipo de investigación trata de comprender la realidad dentro del marco de referencia en el que se encuentra inmersa. Además, Denzin y Lincoln (2000), así como Flick (2002) apuntan que el enfoque cualitativo se interesa por datos que aportan información acerca de una realidad, y que sus resultados son susceptibles transferirse bajo una visión analítica a otros contextos similares.

El presente estudio se sitúa en el noroeste de España y fue desarrollado entre el segundo semestre de 2016 y el primer semestre de 2017. Para ello se ha contado con la participación de 107 padres y madres de 4 colegios Plurilingües que imparten, entre otras, las enseñanzas ordinarias de la educación primaria. Respecto a la infraestructura de los Centros seleccionados, es de indicar que en todos estos colegios, la propia institución cuenta con tabletas digitales para trabajar con el alumnado en el aula, asimismo en el contexto del hogar los niños también disponen de este dispositivo.

Los 107 padres y madres (78 madres y 29 padres) que se han implicado en el presente estudio presentan una media de edad de 42 años, y sus hijos-as cursaban $1^{\circ}, 3^{\circ}, 5^{\circ} \circ 6^{\circ}$ de Educación Primaria. En cuanto a la situación laboral y estudios de estos progenitores, 75 madres y 27 padres estaban en activo; de modo que, solamente tres madres y dos padres se encontraban en situación de desempleo laboral. Sobre su formación escolar, cabe señalar que 11 madres y 5 padres disponen de los estudios básicos, 46 madres y 17 padres tienen estudios secundarios, mientras que 21 madres y 7 padres cuentan con estudios universitarios (tabla 1 ).

\begin{tabular}{cccccc}
\cline { 2 - 5 } Madre & $\begin{array}{c}\text { En } \\
\text { activo }\end{array}$ & $\begin{array}{c}\text { En } \\
\text { paro }\end{array}$ & $\begin{array}{c}\text { Estudios } \\
\text { básicos }\end{array}$ & $\begin{array}{c}\text { Estudios } \\
\text { secundarios }\end{array}$ & $\begin{array}{c}\text { Estudios } \\
\text { universitarios }\end{array}$ \\
\cline { 2 - 6 } Padre & 75 & 3 & 11 & 46 & 21 \\
& 27 & 2 & 5 & 17 & 7
\end{tabular}

Tabla 1. Situación laboral y estudios de los padres y madres del alumnado Fuente: Elaboración propia

Para la recogida de las narrativas se ha diseñado un cuestionario de preguntas abiertas elaborado ad hoc, al no encontrar uno comercializado que se ajustase a las finalidades planteadas en esta 
investigación. En su diseño se ha contado con la colaboración de cuatro expertos, especialistas en el tópico de estudio abordado, que han revisado y asesorado su versión final. De este modo se sostiene la adecuación y pertinencia del mismo. El guion definitivo de preguntas abiertas comprendió, además de los datos de perfil, un total de 13 cuestiones, repartidas en 3 bloques sobre el: Contacto inicial con la tableta; Inclusión curricular de la tableta; e Implicación/colaboración de la familia en la integración académica de la tableta.

Para la recogida de datos, el cuestionario abierto fue entregado en formato papel al profesorado de cada curso en los respectivos colegios, para que por medio del alumnado, se lo hicieran llegar a los padres y madres. En el propio instrumento se informaba de su carácter anónimo y de garantizar la confidencialidad. Al tratarse de un estudio transversal la recogida de esta información se realizó en un momento puntual, a lo largo de una semana, periodo utilizado por los participantes para desarrollar sus narrativas y hacer la entrega al personal investigador, a través de los docentes de sus hijos-as.

En el análisis de las narrativas, inicialmente ha sido necesario organizarlas y enumerarlas consecutivamente, como pasos previos para la aplicación de análisis de contenido. Posteriormente, se ha delimitado el primer nivel de categorización, a partir de los objetivos del estudio, bajo el asesoramiento de los expertos que ya habían participado en la revisión del instrumento. El análisis de contenido de las narrativas se ha realizado con el programa de Analysis of Qualitative Data (AQUAD), versión 7. Lo que ha permitido realizar desarrollar un análisis en profundidad, a partir de una codificación minuciosa que posibilitó generar los resultados teniendo en cuenta el recuento de frecuencias (f). De este modo, en la exposición de los mismos se ofrecen diferentes representaciones icónicas, reflejando la frecuencia y la correspondencia que se establece entre las distintas subcategorías para facilitan su comprensión. Con todo, no se trata de enfatizar su componente cuantitativo, sino de utilizarlo de forma complementaria en la interpretación de resultados. Asimismo, se ilustran con distintos extractos literales que ayudan a anticipar la discusión.

\section{Resultados}

A continuación se presentan los principales resultados estructurados en tres ejes principales que atienden a los bloques de contenido adelantados, así como a las finalidades de investigación planteadas. Sus dimensiones facilitaron la definición de las principales categorías de análisis, asociándolas al: Primer acercamiento de los progenitores a la tableta; Integración curricular del dispositivo; Colaboración escolar de la familia en el uso de este recurso. De ellas derivan las respectivas subcategorías expuestas en adelante.

\section{a. Contacto inicial de la familia con la tableta}

Sobre las dificultades que encontraron, en sus primeros contactos con la tableta, los padres y madres es importante referirse a la formación recibida para su manejo o a si no la consideran necesaria. En este sentido, hay que señalar que los resultados ponen de manifiesto que en general los padres/madres no encuentran dificultades y una minoría refleja que las ha tenido en escasas ocasiones (figura 1). Este hecho puede deberse a la, cada vez, mayor presencia de las TIC en los diferentes contextos sociales y laborales, lo que hace que estos dispositivos pasen también a formar parte de la vida cotidiana de la familia.

Con relación a la formación recibida y/o que pudiesen considerar necesaria para el manejo técnico de la tableta, sorprendentemente, ningún padre-madre estima su necesidad o que haya cantado con algún curso específico. Sobre el menester de disponer de la misma, muy pocos la consideran 
imprescindible y argumentan que es un recurso fácil de manejar y que su utilización es factible de forma autónoma.

Se presentan, a modo de ejemplo, los siguientes extractos:

No me parece complicado usar la tableta digital. Tampoco considero necesario ningún tipo de formación, ya que su uso es muy intuitivo y sencillo (Narrativa no 5: Padre [38 años] de una alumna de $3^{\circ}$ de Primaria; Centro A. Líneas de análisis: 57-61).

No recibí ningún tipo de información, no creo que sea necesaria. Creo que se aprende a base de practicar. En realidad no es difícil (Narrativa no 85: Padre [40 años] de un alumno de $3^{\circ}$ de Primaria; Centro C. Líneas de análisis: 42-48).

La verdad es que no tuve muchas dificultades cuando usé la tableta por primera vez. Lo único que me pareció complicado fue configurarla, pero una vez que la empiezas a utilizar es sencillo. Cuanto más la uso más fácil me parece (Narrativa no 101: Madre [37 años] de una alumna de $1^{\circ}$ de Primaria; Centro D. Líneas de análisis: 39-43).

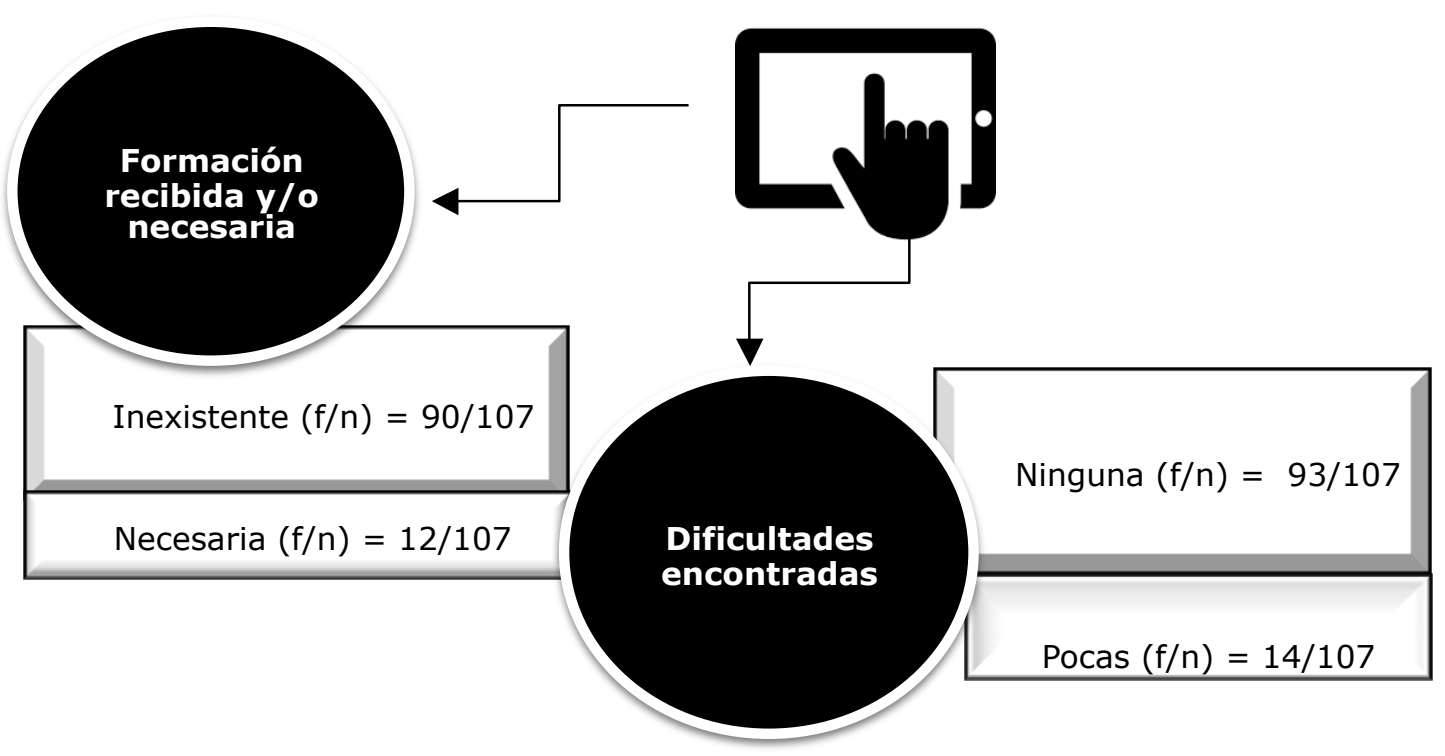

Figura 1. Formación recibida y/o necesaria y dificultades con la tableta Fuente: Elaboración propia

\section{b. Papel de la familia en la integración curricular de la tableta}

Las familias del alumnado están al corriente de las materias del currículum escolar en las que sus hijos-as utilizan la tableta digital. En este sentido identifican su uso en el contexto escolar en las asignaturas de: Ciencias Sociales, Lengua Castellana y Matemáticas (figura 2). Los padres y madres reconocen disponer de este dato fundamentalmente a partir de la información que les facilita el profesorado y de lo que les cuentan sus hijos-as.

En cuanto a la información que tienen los progenitores sobre las actividades que realizan en el aula sus hijos-as, de las narrativas analizadas se desprende que tienen conocimiento de que utilizan la tableta principal para realizar búsquedas de información. Además consideran que muchas de las 
actividades que desarrollan están focalizadas en el empleo de juegos didácticos (Trivial [infantil], Increíble Chapuzón de Zoe, Mounstruario, etc.) y, en menor medida, en aplicaciones audiovisuales. Nuevamente, aseguran que esta información se la proporcionada el profesorado (con el que mantienen una relación próxima) y sus propios hijos-as.

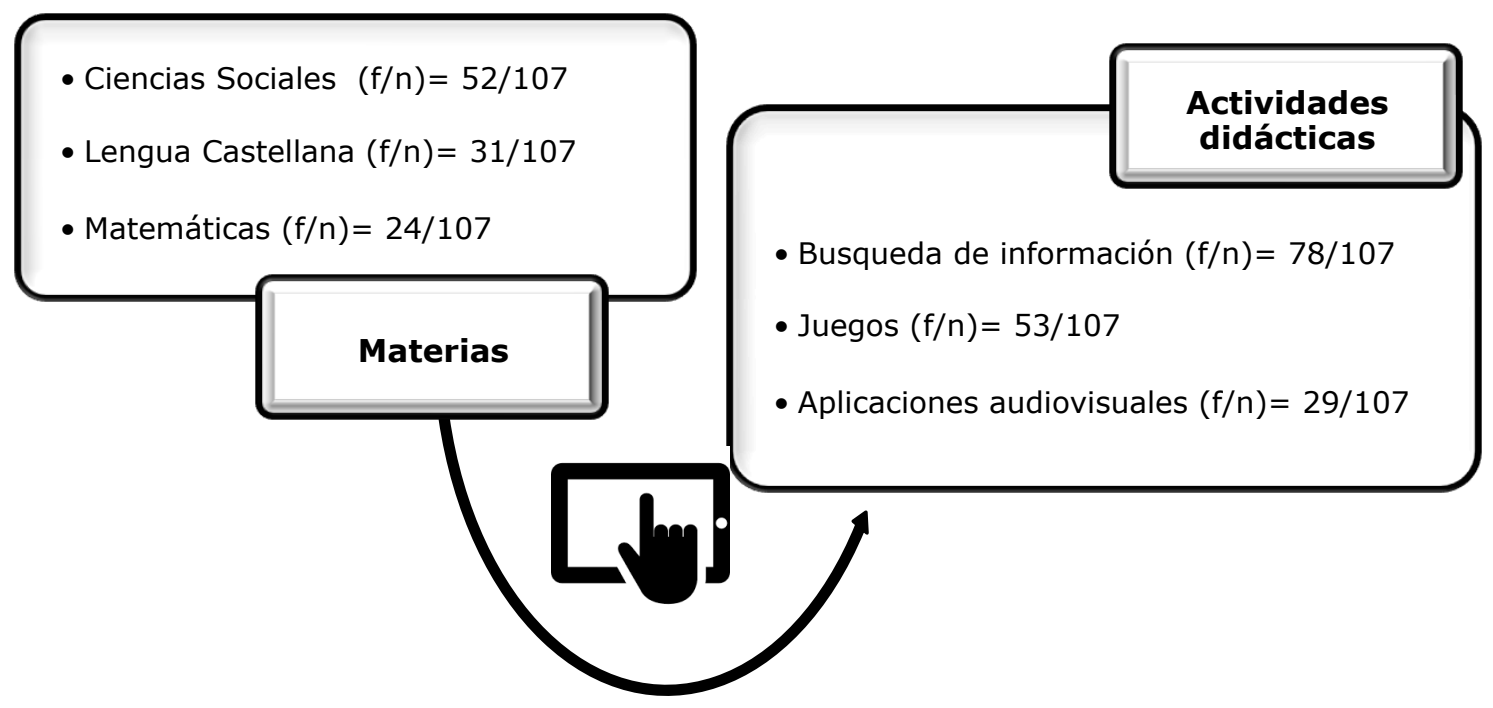

Figura 2. Materias y actividades que los niños-as desarrollan con la tableta en el aula Fuente: Elaboración propia

De modo ilustrativo se presentan los siguientes extractos de las narrativas de los progenitores:

Mi hijo me cuenta que utilizan la tableta sobre todo en Ciencias Sociales y Matemáticas. En cuanto a las actividades que hacen suelen ser para buscar información, jugar... (Narrativa no 105: padre [43 años] de un alumno de $5^{\circ}$ de Primaria; Centro D. Líneas de análisis: 6872).

Me tiene hablado de que realizan vídeos o montajes de fotos, también buscan información sobre autores y la utilizan para hacer trabajos. Su profesor también nos informa en las reuniones sobre las actividades que realizan en el aula (Narrativa no 53: madre [43 años] de un alumno de $6^{\circ}$ de Primaria; Centro B. Líneas de análisis: 78-83).

Sé que las usan en Ciencias Sociales, Matemáticas y Lengua Castellana. El otro día me contó mi hija que hicieron una actividad que consistía en localizar las provincias de España en un mapa interactivo. Venía muy contenta porque se divirtió mucho y me consta que al mismo tiempo aprendió (Narrativa no 2: madre [40 años] de una alumna de $3^{\circ}$ de Primaria; Centro A. Líneas de análisis: 65-73).

La motivación del alumnado es un aspecto fundamental y sus progenitores lo identifican con facilidad. Al respecto, el colectivo de los padres y madres participantes coincide, de modo acentuado, en que la inclusión de la tableta digital en el contexto escolar supone un incentivo valioso para sus hijos-as en el aprendizaje (figura 3 ). Muy pocos padres-madres consideran que la motivación de su hijo-a no aumenta al incluir este recurso en el proceso formativo. Sin embargo, 
sorprendentemente sobre la percepción de mejora del rendimiento académico no existe un consenso y 54 padres-madres sostienen que las tabletas en el aula no contribuyen a un mejor rendimiento, mientras que 53 opinan que sí existe. A pesar de esta discrepancia específica, la valoración global positiva.

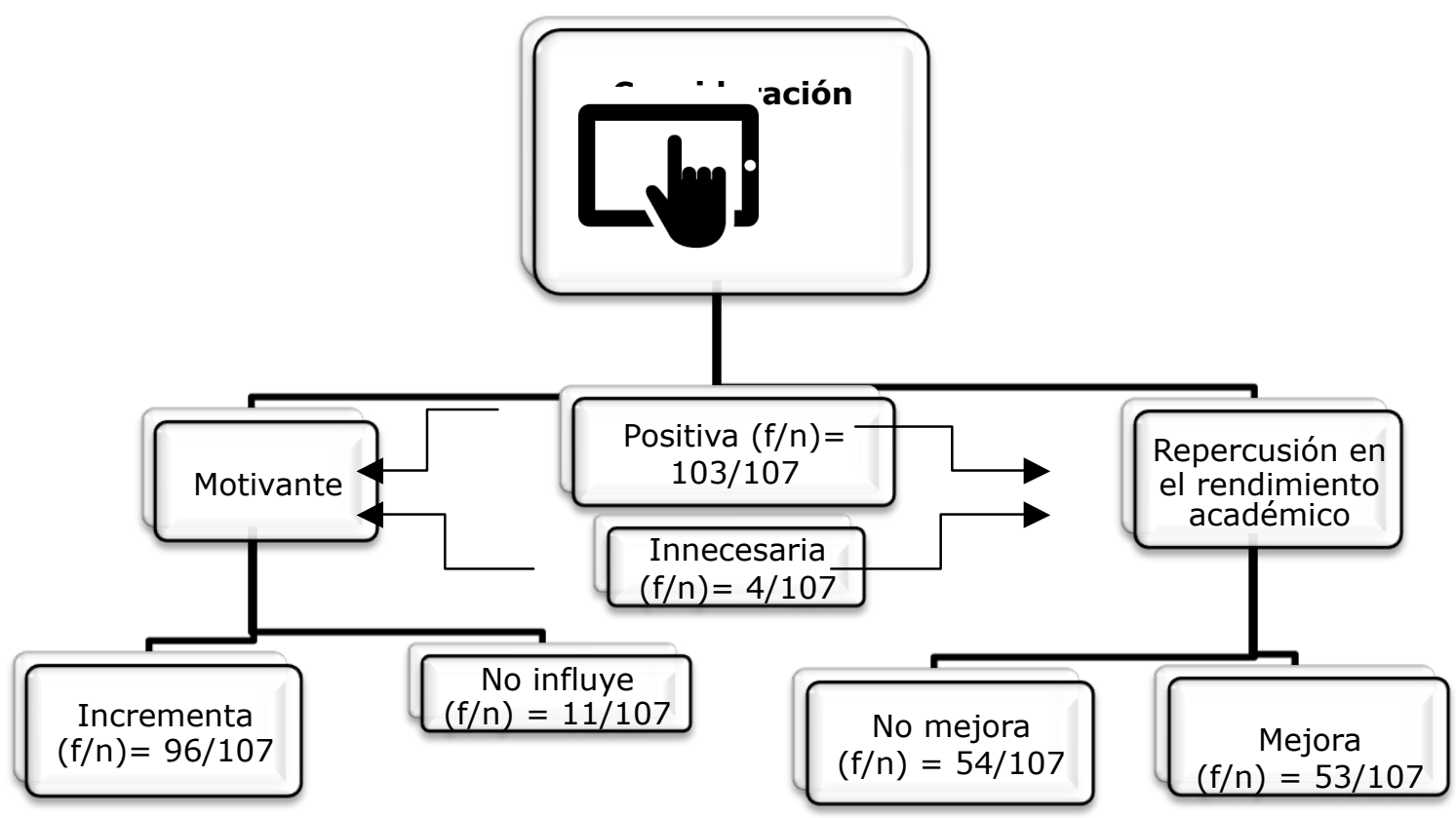

Figura 3. Consideración de los progenitores sobre el uso de la tableta Fuente: Elaboración propia

A modo de ejemplo se exponen los siguientes extractos ilustrativos:

Mi valoración sobre la inclusión de la tableta en el aula es globalmente positiva. Creo que es un recurso que les motiva mucho y eso siempre es interesante aunque, en mi caso, no encuentro mejoras en el rendimiento de mi hija. A decir verdad, siempre fue una niña muy responsable y con muy buen rendimiento académico, o me puedo quejar (Narrativa no 102: Madre [41 años] de una alumna de $5^{\circ}$ de Primaria; Centro D. Líneas de análisis: $72-$ 78).

Valoro positivamente que utilicen las tabletas digitales en el centro. Las Nuevas Tecnologías son el futuro y deben saber utilizarlas. Además mi hijo está mucho más contento y motivado desde que la usa. Creo que es un incentivo muy bueno y que ayuda a que trabajen más (Narrativa no 61: Padre [43 años] de un alumno de $6^{\circ}$ de Primaria; Centro B. Líneas de análisis: 59-63).

Creo que la incorporación de las tabletas digitales al aula fue un acierto. Mi valoración es totalmente positiva. Me parece una forma de aprender muy divertida y muy motivadora para los niños-as. Personalmente, pienso que mi hija mejoró su rendimiento académico desde que trabaja con las tabletas (Narrativa no 7: Madre [37años] de una alumna de $3^{\circ}$ de Primaria; Centro A. Líneas de análisis: 62-69). 
Otro aspecto interesante para conocer la percepción de las familias sobre la inclusión de la tableta digital en el aula es el relacionado con las reuniones/ información que se les proporciona desde el centro (figura 4). En este sentido, un grupo amplio de padres-madres afirman que han sido convocados a una reunión para abordar la incorporación de la tableta al proceso de enseñanzaaprendizaje de sus hijos-as, mientras que una parte más reducido del colectivo sostiene lo contrario. Cabe destacar que esta diferencia puede ser provocada por la interpretación de cada padre-madre realiza acerca de la información recibida, ya que por los datos recogidos, en vía documental (actas de reuniones y circulares informativas de los centros), se corrobora que en los 4 centros se ofreció, como mínimo, una reunión inicial con el fin de comunicar la incorporación de la tabletas en el proceso de aprendizaje de los niños-as.

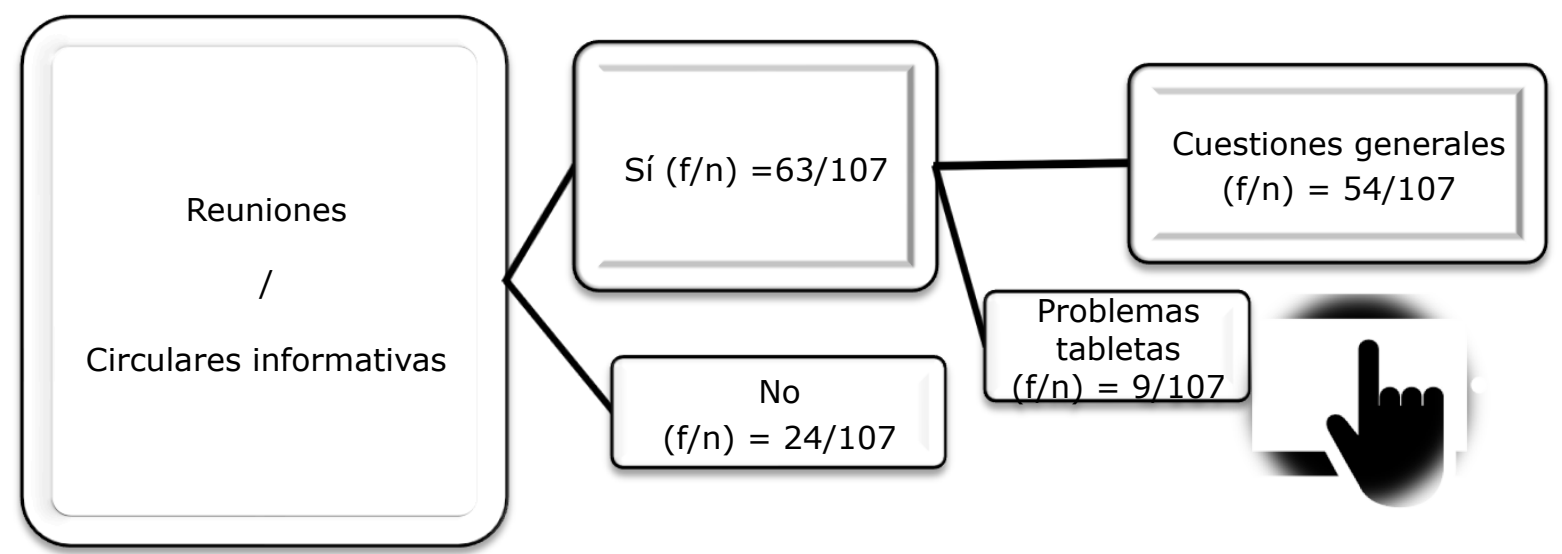

Figura 4. Reunión/información recibida sobre la inclusión curricular de la tableta Fuente: Elaboración propia

Un grupo amplio de progenitores, hacen alusión a que se le convoca a reuniones en el Centro, tanto a inicios de curso, como de forma periódica sucesivamente, afirmando que en ellas no se profundiza en cuestiones relacionadas con la inclusión de la tableta en el aula. Con todo, 9 padresmadres concretan que en dichos encuentros se trataron colateralmente problemáticas sobre el mal funcionamiento de alguna aplicación educativa y las dificultades técnicas para el manejo del dispositivos digitales, con el fin de buscar soluciones para disfrutar de los beneficios que aportan estos recursos a la educación de sus hijos-as.

Se aportan algunos extractos de modo ilustrativo:

No recuerdo la existencia de ninguna reunión específica para tratar la inclusión de las tabletas digitales. Sí es verdad que a principio de curso tuvimos la reunión como todos los años y se comentó que nuestros hijos-as iban a trabajar con esos recursos (Narrativa no 2: Padre [37 años] de un alumno de $1^{\circ}$ de Primaria; Centro A. Líneas de análisis: 106-111).

Tuvimos una reunión a principio de curso con el profesor tutor, y entre otras cosas nos explicó que este curso iban a incorporar un nuevo recurso, refiriéndose a la tableta digital (Narrativa no 81: Madre [39 años] de una alumna de $3^{\circ}$ de Primaria; Centro C. Líneas de análisis: 103-110). 


\section{c. Participación de los progenitores en el uso de la tableta}

Un aspecto objeto de análisis ha sido la utilización que realizan los padres y madres con la tableta digital con sus hijos-as, en el contexto del hogar. En este sentido, cabe destacar que la principal actividad que realiza la familia con sus hijos-as es la búsqueda de información; tanto para desarrollar las tareas encomendadas desde el centro como para búsquedas libres de información o recursos diversos (figura 5). También dedican tiempo a los juegos interactivos y en menor medida a escuchar música y al visionado de películas.

En general en el contexto del hogar los padres-madres suelen desarrollar las prácticas con la tableta, fundamentalmente, los fines de semana o en períodos festivos. Cabe señalar que, desafortunadamente, es infrecuente el uso de la tableta para fomentar hábitos lectores, a excepción de 3 padres, que no la utilizan para realizar ningún tipo de actividad de este tipo.

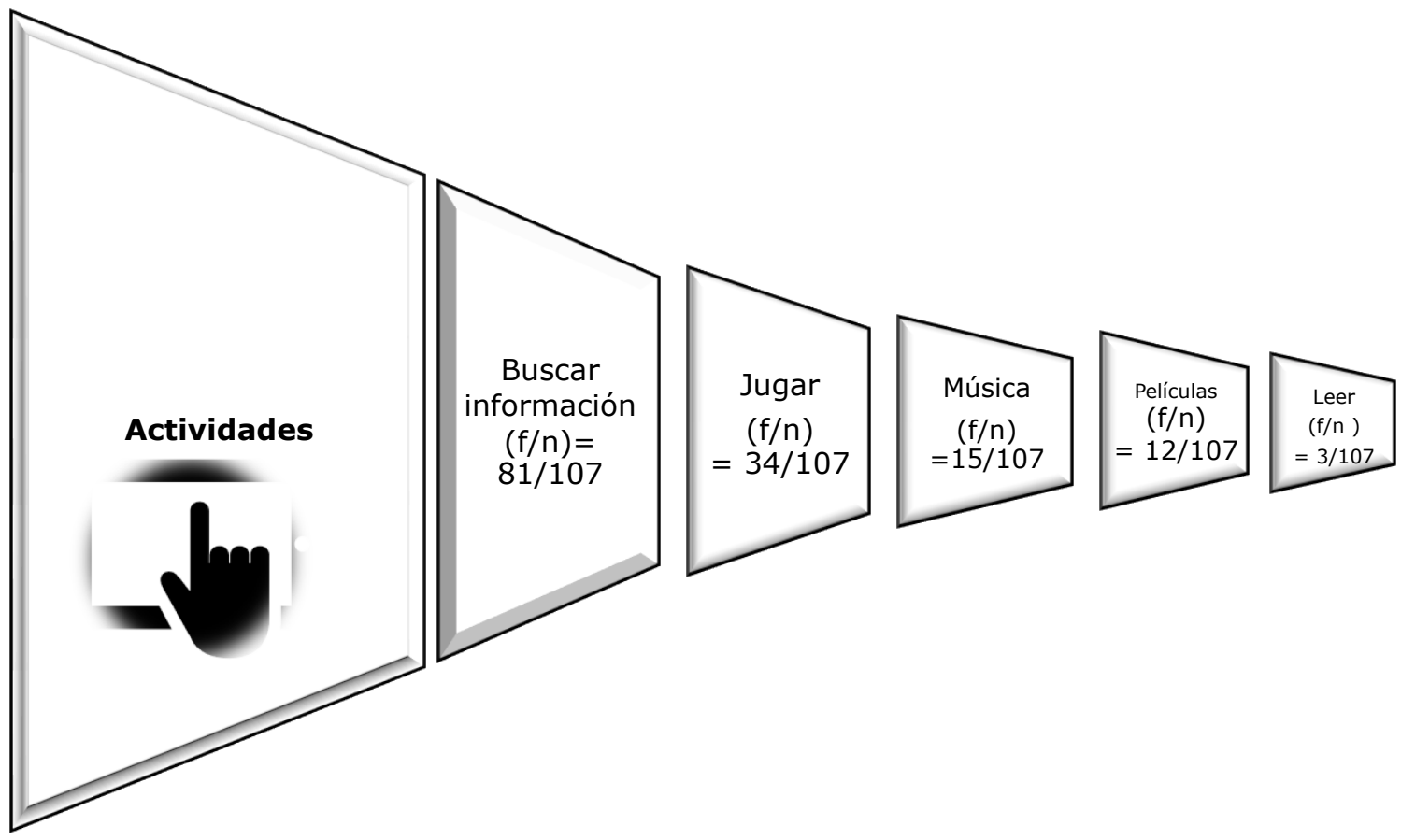

Figura 5. Actividades desarrolladas con la tableta en el contexto familiar Fuente: Elaboración propia

Se presentan los siguientes extractos a modo de ejemplo:

Sí, en casa la utilizamos mucho, sobre todo el fin de semana o en vacaciones. Por la semana sólo la usamos para buscar información que necesita para los deberes o para algún trabajo (Narrativa no 99: Padre [41 años] de una alumna de $3^{\circ}$ de Primaria; Centro D. Líneas de análisis: 128-132).

La usa bastante, más de lo que me gustaría pero soy consciente de que hoy en día es un recursos necesario. Recurre a ella para buscar información tanto para el colegio como de interés personal y los fines de semana escuchamos música, vemos alguna película o jugamos a algún juego que le guste (Narrativa no 11: Madre [39 años] de una alumna de $5^{\circ}$ de Primaria; Centro A. Líneas de análisis: 113-119). 
La usamos sobre todo para buscar información. En el colegio le mandan muchos trabajos en los que tienen que buscar la vida de algún autor, sus principales obras...y luego las ponen en común en clase. Hoy en día hay mucha información en Internet, aunque no toda es fiable por eso muchas veces la busco con ella para que distinga la información buena de la que no lo es (Narrativa no 83: Madre [38 años] de una alumna de $3^{\circ}$ de Primaria; Centro C. Líneas de análisis: 119-124).

En cuanto a la forma en que los-as padres-madres animan o incitan a sus hijos-as a utilizar la tableta, de forma notoria, sostienen que no es necesario incentivarles (figura 6). En este sentido, no queda claro que los padres-madres logren comprender que el uso lúdico de la tableta también puede contener aportes educativos de gran interés para la formación académica de los menores. Los progenitores que promueven en sus hijos-as la utilización de este dispositivo lo hacen, o bien para solucionar dudas, o para buscar aplicaciones de interés educativo.

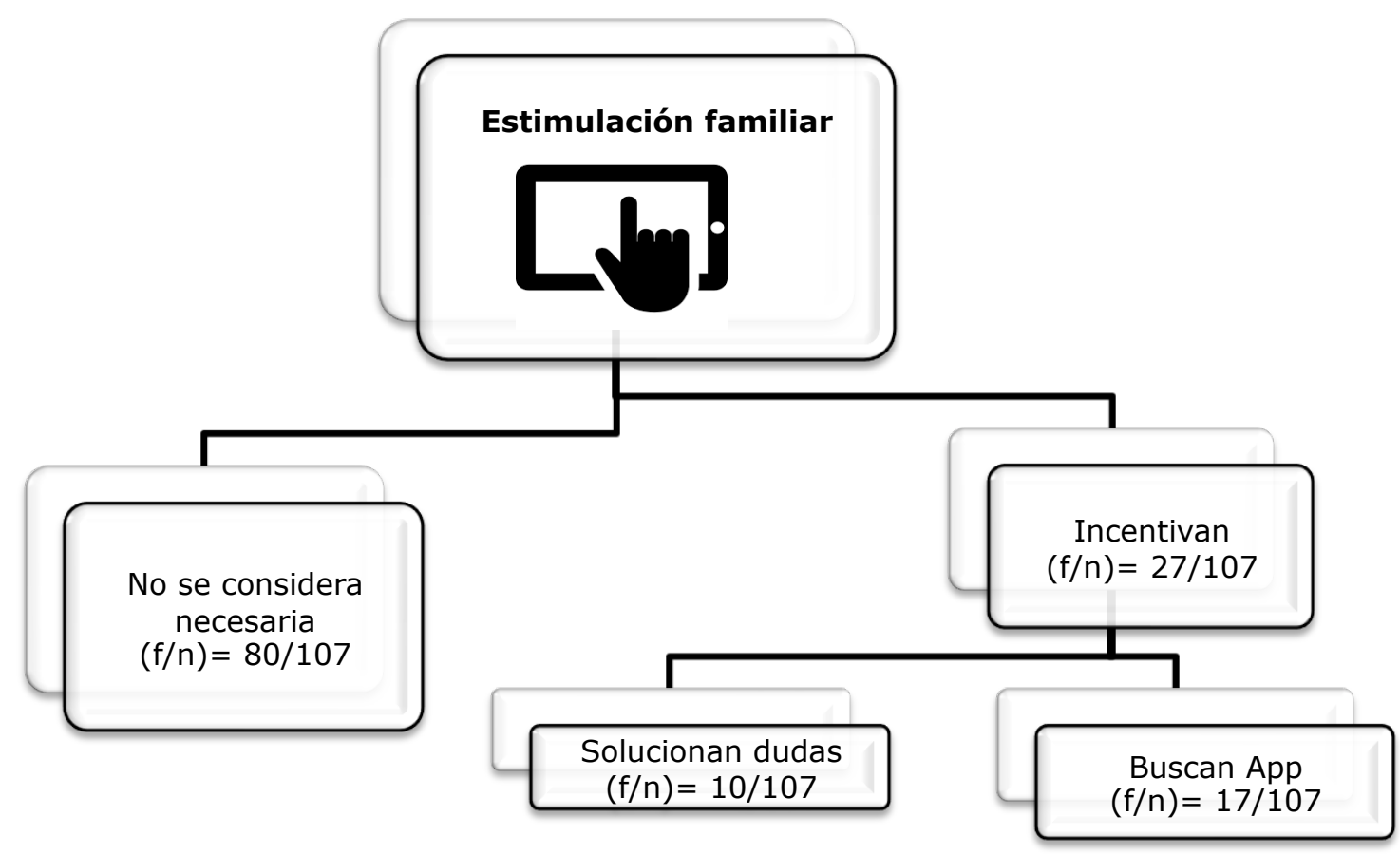

Figura 6. Estimulación potenciada por la familia a partir de la tableta Fuente: Elaboración propia

Véanse los siguientes extractos ilustrativos:

En casa no la animamos porque creemos que ya está bastante animada ya que no hace falta mandarle coger la tableta, la coge ella sola (Narrativa no 100: Madre [38 años]de una alumna de $5^{\circ}$ de Primaria; Centro D. Líneas de análisis: 140-146).

Lo animamos en todo, en cuanto a la tableta le decimos que busque información para solucionar las dudas que tenga, ya que hoy en día la información está al alcance de la mano, eso sí con mucho cuidado que no toda la información es fiable y valiosa (Narrativa no 4: Madre [38 años] de una alumna de $3^{\circ}$ de Primaria; Centro A. Líneas de análisis: $120-$ 131). 
Sí que la animo porque considero que es un recurso muy útil. Le ayudo a buscar aplicaciones educativas interesantes que le puedan servir para repasar lo aprendido en el colegio (Narrativa no 63: Padre [44 años] de una alumna de 60 de Primaria; Centro B. Líneas de análisis: 115-118).

\section{Discusión y conclusiones}

Las nuevas tecnologías se adentraron con fuerza en los hogares españoles y lo han hecho para permanecer y continuar e incrementar su utilización en el futuro. En general la actividad con las TIC se encuentra integrada en la cotidianidad de la vida familiar. Tanto en los hogares, como en los centros educativos se cuenta con dispositivos diversos y, cada vez en mayor medida, con tabletas digitales, aunque su utilización didáctica en las escuelas no se encuentra normalizada (Ebner, Schönhart y Schön, 2014; Marés, 2012). Con todo, los principales entornos de interacción del alumnado con las TIC se producen en el espacio del centro educativo y en el hogar. De ahí la importancia de implicar a la familia ante la tenue atención al uso de las tabletas en el contexto del hogar. De hecho Valdés, Urías, Wendlandt y Torres (2014) sostienen que la participación de la familia en la educación comprende la implicación de los progenitores en actividades que favorezcan en los estudiantes la adquisición de competencias demandadas por el currículo escolar, entre ellas la digital.

De este trabajo se desprende que desde los progenitores no se perciben inconvenientes relevantes para el manejo de los dispositivos digitales, así mismo afirman que no tienen dificultades técnicas a la hora de usar la tableta digital. Tampoco recibieron formación específica, pero no la consideran necesaria. Otros estudios (Torrecillas-Lacave, Vázquez-Barrio y Monteagudo-Barandalla, 2017) también han determinado que los padres-madres no suelen tener dificultades en el manejo de los dispositivos digitales. Por otra parte, cabe traer a colación la importancia de ofrecer formación especializada a los profesionales de la educación (educadores-as, profesorado, orientadores, etc.), con el fin de capacitarles para planificar e implementar modelos educativos apoyados en las TIC (Marqués, 2014; Macià Bordalba y Garreta Bochaca, 2018), y poder proporcionar un adecuado asesoramiento a las familias. Con este estudio se pone de manifiesto que los progenitores conocen las materias curriculares en las que trabajan sus hijos-as en el aula con la tableta, en concreto identifican las de Ciencias Sociales, Lengua Castellana y Matemáticas. También son conocedores de las actividades que realizan y de que les motivan y divierten. En este sentido, reconocen que utilizan la tableta principalmente para la búsqueda de información, el uso de distintas aplicaciones audiovisuales, el visionado de películas, juegos o para escuchar de música. Asimismo consideran, de forma notoria, que el uso de este dispositivo, tanto en la escuela, como en el hogar, incrementa la motivación de los niños-as y, en general, su postura respecto a la utilización de este recurso es muy positiva.

Es de señalar que respecto a la mejora producida por la tableta en el rendimiento académico no se puede asegurar, al encontrarse dividida la opinión de los padres-madres. En este sentido también en otros estudios se ha puesto de manifiesto esta discrepancia. Por ejemplo, Green (2016) afirma que el uso de las tabletas digitales mejora el rendimiento del alumnado, mientras que De Witte y Rogge, 2014) ponen de manifiesto que en general las TIC no produce, por sí misma, una mejora en el rendimiento de los estudiantes. Como beneficios globales, Hye y Hwan (2015), Sackstein, Spark y Jenkins (2015), así como Viñas y Cabezuelo (2012) apuntan que el uso de las nuevas 
tecnologías, entre ellas la tableta digital, acarrea efectos muy positivos para el alumnado (abren la posibilidad de consumir contenidos de manera no lineal, se realizan aprendizajes más innovadores, son recursos idóneos para la lectura y para mejorar la capacidad de comprensión, etc.). Asimismo, algún autor afirma que la tableta fomenta la apropiación efectiva de esta tecnología, fortalece las dinámicas del aprendizaje, la inclusión social y genera más y mejores oportunidades (Merchán y Mesa, 2018).

El uso de la tableta por el alumnado de Educación Primaria en el hogar, para utilizar como ayuda en el desarrollo de las actividades/trabajo de aula, todavía no se encuentra normalizado. Incluso, lo más común es que los padres-madres no incentiven a sus hijos-as a utilizarla, al considerar que la emplean suficientemente en el centro educativo. Por esta razón, sostienen que no es necesario animar a sus pequeños-as para que la utilicen. De todas formas, existen excepciones ya que algunos progenitores incentivan a sus hijos-as para trabajar con este dispositivo, al estimar que es un recurso muy interesante y de mucha utilidad, tanto actualmente como de cara al futuro. Cabe señalar, al respecto, que coincidimos con Ballesta y Cerezo (2011), en que para abordar la integración de las TIC en los centros educativos es imprescindible contar con la implicación de la familia. Por otra parte hay que resaltar la relevancia de la alfabetización digital, al favorecer el uso seguro y crítico de la propia tecnologías en diferentes ámbitos: formativo, desarrollo personal, participación ciudadana, etc. (Martínez Bravo, Sádaba y Serrano (2018).

La familia juega un papel muy importante a la hora de garantizar que sus hijos-as realicen un uso apropiado de la tecnología. Por ello, es de señalar que los progenitores deben ayudar a sus hijos-as a descubrir los aspectos positivos y negativos de la misma, y le establezcan normas para un uso apropiado, seguro y responsable (Ruíz, Rodríguez y Alaminos, 2012; San Sebastián, Quintero, Correas, De Dios y Echániz, 2011). Por este motivo la educación digital del mañana dependerá, en buena medida, de la acción de las familias y de la escuela, siendo necesaria la colaboración entre ambas (Gabelas y Marta, 2008; Bartau-Rojas, Aierbe-Barandiaran y Oregui-González, 2018). Tal como se desprende de este estudio es necesario continuar trabajando en este sentido para descubrir el posicionamiento de los progenitores ante el uso de los dispositivos digitales, y en particular de la tableta, para articular e incorporar estrategias que contribuyan a mejorar la situación actual.

\section{Agradecimientos}

Este estudio forma parte de la Tesis Doctoral sobre el uso de las tabletas con el alumnado de Educación Primaria, y aprovechamos para manifestar el agradecimiento a los participantes e instituciones implicadas. 


\section{Referencias}

Aguilar, M. y Leiva, J. (2012). La participación de las familias en las escuelas TIC: análisis y reflexiones educativas. Pixel-Bit. Revista de Medios y Educación, 40, 7-19. Recuperado de goo.gl/XXFgya

Arias-Ortiz, E. y Cristia, J. (2014). El BID y la tecnología para mejorar el aprendizaje: ¿Cómo promover programas efectivos? Recuperado de goo.gl/rmYJII

Balcázar, P., González, N. Gurrola, G. y Moysén, A. (2015). Investigación cualitativa. México: Universidad Autónoma del Estado de México.

Ballesta, F. y Cerezo, M. C. (2011). Familia y escuela ante la incorporación de las tecnologías de la información y la comunicación. Educación XX1, 14(2), 133-156.

Bartau-Rojas, I., Aierbe-Barandiaran, A. y Oregui-González, E. (2018). Mediación parental del uso de Internet en el alumnado de Primaria: creencias, estrategias y dificultades. Comunicar, 54, 71-79.

Cabrera, M. (2009). La importancia de la colaboración familia-escuela en la educación. Innovación y Experiencias Educativas, 16, 1-9. Recuperado de http://www.csicsif.es/andalucia/modules/mod_ense/revista/pdf/Numero_16/MARIA_CABRERA_1.pd f

Campos-Domínguez, E. y Redondo-García, M. (2015). Meta periodismo y transparencia informativa en el periodismo del siglo XXI. OBETS. Revista de Ciencias Sociales, 10(1), 185-209.

Collet, J. y Tort, A. (2013). Escuelas, familias y éxito escolar para todos/as. Mejorar los vínculos entre docentes y familias, ¿mejora los resultados académicos? Cuadernos de Pedagogía, 428, 82-85.

Cortés, S., Méndez, L. y Lacasa, P. (2016). Ipads, Apps y Redes Sociales. Construyendo narrativas multimodales en las aulas. Digital Education Review, 30, 53-75. Recuperado de http://revistes.ub.edu/index.php/der/article/view/16120/pdf

De Witte, K. y Rogge, N. (2014). Does ICT matter for effectiveness and efficiency in mathematics education? Computers \& Education, 75, 173-184.

Denzin, N. y Lincoln, Y. (Eds.) (2000). Handbook of Qualitative Research. Londres: Sage

Ebner, M., Schönhart, J. y Schön, S. (2014). Experiences with Ipads in primary school. Profesorado. Revista de Currículum y Formación del Profesorado, 18(3), 161-173.

Espiritusanto, O. (2017). Generación Z: móviles, redes y contenido generado por el usuario. Revista de Estudios de Juventud, 114, 111-126.

Estaban-Moreno, R. M. (2017). Tecnologías, educación y brecha digital. Tendencias pedagógicas, 29, 5-6.

Feliz-Murias, T., Ricoy, C. y Feliz, S. (2014). Innovative didactical strategies based on tablets and u-learning. En Actas de la conference proceedings the open and flexible higher education conference (pp. 142156). Heerlen: EADTU. Recuperado de http://conference.eadtu.eu/images/Proceedings/Conference_2014_-_proceedings.pdf

Fernández-Rodrigo, L. y Carrera, X. (2016). Aspectos organizativos y técnicos para la integración de tabletas digitales en centros de Educación Primaria y Secundaria de Cataluña. EDUTEC. Revista Electrónica de Tecnología Educativa, 58, 1-14.

Ferrer, V. (2017). Nueva tecnologías, competencia y procesos de aprendizaje: La experiencia del WMCP y la Valencianada. ReVisión, 10(2), 79-83.

Flick, U. (2002). Introducción a la investigación cualitativa. Madrid: Morata.

Gabelas, J. A. y Marta, C. (2008). Consumos y mediaciones de familias y pantallas. Nuevos modelos y propuestas de convivencia. Zaragoza: Gobierno de Aragón.

García, M., Ferrás, C., Rocha, A. y Aguilera, A. (2016). Mobile phones and psychosocial therapies with vulnerable people: A first state of the art. Journal Medical Systems, 40, 157-170.

García-Rodríguez, M. y Paíno, N. (2017). Coordinación educativa escuela-familia. Dificultades y soluciones. Revista de Estudios e Investigación en Psicología y Educación, Vol. Extr(5), 75-78.

Green, A. (2016). Significant returns in engagement and performance with a free teaching App. Journal of Economic Education, 47(1), 1-10. 
Hargreaves, A. y Fullan, M. (2012). Professional capital: Transforming teaching in every school. Nueva York: Teachers College Press.

Hernández, R. (2017). Impacto de las TIC en la educación: Retos y Perspectivas. Propósitos y Representaciones, 5(1), 325-347.

Hye, K. y Hwan, J. (2015). Factors influencing studen's beliefs about the future in the context of tablet-bassed interactive classrooms. Computers \& Education. 89, 1-15.

INE (2017). Encuesta sobre equipamiento y uso de tecnologías de información y comunicación en los hogares. Madrid: INE. Recuperado de goo.gl/g9d2cb

Livingstone, S., Haddon, L., Görzig, A. y Ólafsson, K. (2011). EU kids online II: Final report. Recuperado de http://eprints.Ise.ac.uk/39351

López-González, R. y Anaya, R. (2016). Estudiantes universitarios interactuando en red: ¿nuevos escenarios de interacción, expresión y participación ciudadana? Revista Interamericana de Educación de Adulto, $38(1), 48-67$.

Lozano, J., Alaraz, S. y Colás, P. (2013). Los centros educativos multiculturales y sus relaciones con las familias. Revista Educación XXI, 16(1), 210-232.

Macià Bordalba, M. y Garreta Bochaca, J. (2018). Accesibilidad y alfabetización digital: barreras para la integración de las TIC en la comunicación familia/escuela. Revista de Investigación Educativa, 36(1), 239-257.

Marés, L. (2012). Tablets en educación. Oportunidades y desafíos en políticas uno a uno. Recuperado de www.oei.es/70cd/Tabletseneducacion.pdf

Marquès, P. (2014). Metainvestigación 2013-14. Uso educativo de las tabletas digitales en educación infantil. Informe final. Recuperado de http://es.slideshare.net/peremarques/uso-educativo-delas-tabletasdigitales-en-educacin-infantil

Martín, M., Tedesco, J. C., López, J. A., Acevedo, J. A., Echeverría, J. y Osorio, C. (2009). Educación, ciencia, tecnología y sociedad. Madrid: Centro de Altos Estudios Universitarios de la OEI.

Martínez Bravo, Sádaba, Ch. y Serrano, J. (2018). Desarrollo de competencias digitales en comunidades virtuales: un análisis de "ScolarTIC". Prisma Social: Revista de Investigación Social, 20, 129159. Mascheroni, G. y Kjartan, O. (2014). Net children go mobile. Risks and opportunities. Recuperado de http://goo.gl/a7CB5H

Merchán, L. y Mesa, F. Y. (2018). Políticas de aprendizaje móvil en el ámbito colombiano. Boletín Redipe, 7(3), 90-97.

Morgado, B. y Román, M. (2011). La familia como contexto de desarrollo infantil. En V. Muñoz (Eds.), Manual de psicología del desarrollo aplicada a la educación (pp. 37-60). Madrid: Ediciones Pirámide.

Ricoy, M. C. y Feliz, T. (2002). Estrategias de intervención para la escuela de padres y madres. Educación XXI, 5, 171-197.

Ruíz, B., Rodríguez, A. y Alaminos, F. J. (2012). Tecnologías de la Información y Comunicación. Serie: Estado de la infancia y adolescencia en Andalucía. Granada: Observatorio de la Infancia en Andalucía.

Sackastein, S., Suzanne, S. y Jenkins, A. (2015). Are e-books effective tolos for learning? Reading speed and comprehension: iPad (R)(i) vs. Paper. South African Journal of Education, 35(4), 1-14.

San Sebastián, J., Quintero, J., Correas, J., De Dios, M. J. y Echániz, T. (2011). Patrones de uso, abuso y dependencia a las tecnologías de la información en menores (Internet, móvil, videojuegos, TV). Madrid: CONFIAS.

Sánchez, I. y Cortada, M. (2015). Recursos digitales en la relación familia y escuela en la etapa 0-3. Cultura y Educación, 27(1), 221-233.

Sánchez-Martínez, C. y Ricoy, M. C. (2015). El impacto de la tableta en la educación primaria. Revista de Estudios e Investigación en Psicología y Educación, 13, 93-97. 
Torrecillas-Lacave, T., Vázquez-Barrio, T. y Monteagudo-Barandalla, L. (2017). Percepción de los padres sobre el empoderamiento digital de las familias en hogares hiperconectados. El Profesional de la Información, 26(1), 97-104.

Tuzel, S. y Hobbs, R. (2017). El uso de las redes sociales y la cultura popular para una mejor comprensión intercultural. Comunicar, 51, 63-72. Recuperado de https://doi.org/10.3916/C51-2017-06s

Urías-Martínez, M., Urías-Murrieta, M. y Valdés-Cuervo, A. (2017). Creencias docentes del uso de tecnologías por familias para involucrarse en educación. Apertura, 9(2), 148-159.

Valdés, Á., Urías, M., Wendlandt, T. y Torres G. (2014). Relation between family dynamics and teachers' practices with the participation of mothers in the education of the children. Journal of Education and Human Development, 3(3), 309-315.

Viñas, M. y Cabezuelo, F. (2012). La iPad de Apple como herramienta para la formación en competencias digitales de los estudiantes de periodismo. Sevilla: Universidad de Sevilla. Recuperado de http://www.gabinetecomunicacionyeducacion.com/files/ 